\title{
Near-coast tsunami waveguiding: phenomenon and simulations
}

\author{
E. van Groesen ${ }^{1,2}$, D. Adytia ${ }^{2}$, and Andonowati ${ }^{1,2,3}$ \\ ${ }^{1}$ Department of Applied Mathematics, University of Twente, The Netherlands \\ ${ }^{2}$ LabMath-Indonesia, Bandung, Indonesia \\ ${ }^{3}$ Department of Mathematics, Institut Teknologi Bandung, Indonesia
}

Received: 5 November 2007 - Revised: 31 January 2008 - Accepted: 3 February 2008 - Published: 3 March 2008

\begin{abstract}
In this paper we show that shallow, elongated parts in a sloping bottom toward the coast will act as a waveguide and lead to large enhanced wave amplification for tsunami waves. Since this is even the case for narrow shallow regions, near-coast tsunami waveguiding may contribute to an explanation that tsunami heights and coastal effects as observed in reality show such high variability along the coastline. For accurate simulations, the complicated flow near the waveguide has to be resolved accurately, and grids that are too coarse will greatly underestimate the effects. We will present some results of extensive simulations using shallow water and a linear dispersive Variational Boussinesq model.
\end{abstract}

\section{Introduction}

Tsunami heights and effects show a high variability along the coast, as has been observed in numerous field surveys that are reported in various journals and on websites (EERI (2005), Liu et al., 2005, Borrero et al., 2006, Synolakis and Kong, 2006, Reese et al., 2007). Numerical simulations of the effect of tsunamis on the coast are difficult and still rather inaccurate. One reason is the uncertainties of the seafloor deformations that determine the tsunami initialization. Another major reason is the poor modeling of the incoming wave in the near-shore region and the run-up on the land. Details of bathymetry, land-topography and land characteristics have important effects on the run-up and inundation results, see for instance Yeh et al. (1993), Titov and Synolakis (1997) and Fritz et al. (2007). A good overview by Synolakis and Bernard (2006) describes how various aspects of tsunami science developed over the past decades, and Chatenoux and Peduzzi (2005) report about the difficulties encountered in the coastal run-up modeling. Numerical simulations with a grid-

Correspondence to: E. van Groesen

(groesen@math.utwente.nl) size determined by the gridsize with which the bathymetry is known (sometimes as coarse as one-minute), seem not to able to capture the high coastal variability, and often seem to underestimate the most extreme heights that are observed in the field.

This paper aims to increase our understanding by emphasizing one aspect that may contribute to high variability along the coast. We will show that relatively narrow and shallow elongated areas in the propagation direction can produce largely amplified waves by a process that we will call nearcoast waveguiding. The larger wave heights and large variations over relatively small transversal distances in the nearcoast area will cause corresponding large variability on the shoreline, leading to local differences in the run-up properties.

Near-coast tsunami waveguiding should be distinguished from two other well-known types of waveguiding. The first one is when waves from the ocean are trapped by the continental shelf to form so-called edge waves. The continental shelf is parallel to the shoreline and being shallower it acts as waveguide and traps waves that will run in an alongshore direction with a profile that is decaying towards the deeper ocean; see for instance Kajiura (1970), Gonzalez et al. (1995), Fujima et al. (2000) and Kurkin and Pelinovsky (2002). Waveguiding above ocean ridges appears when waves are trapped by the waveguide formed by the ridge that is shallower than the surrounding ocean bottom; see for instance Titov et al. (2005). The near-coast waveguiding in this paper is similar to this last one, now with the waveguided ridge directed from the ocean toward the coast, more or less perpendicular to the shoreline. Then waveguiding takes place for incoming waves from the ocean approaching the coast, very different from the alongshore edge waves; see also Ishii and Abe (1980).

The near-coast tsunami waveguiding phenomenon is caused by the fact that different propagation speeds of waves between deeper and shallower parts in the bathymetry give

Published by Copernicus Publications on behalf of the European Geosciences Union. 


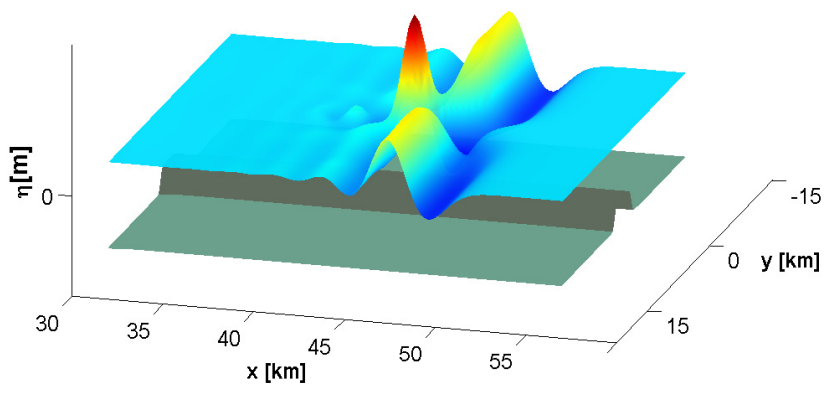

Fig. 1. Snapshot of the surface elevation showing the characteristic tsunami waveguiding phenomenon. The incoming wave from the left is amplified and delayed above a ridge that acts as waveguide.

rise to characteristic distortions of the surface elevation near the waveguide. Inside the waveguide the wave will have a largely amplified wave height. But outside the waveguide the distorted wave has to adjust to the finite amplitude undisturbed external wave, which will influence both the profile and the velocity of guided tsunami waves, and which causes the transversal variability. We will show the appearance of guided waves with largely amplified amplitudes as illustrated in Fig. 1 for the case of a specific geometry with synthetic bathymetry, as illustrated in Fig. 2. In a forthcoming paper we will show that bathymetries leading to such phenomena actually appear for specific parts of the Indonesian coast. In another paper we will derive a low dimensional dynamical model that describes the phenomenon theoretically.

In this paper we show some results of numerical simulations above a synthetic bathymetry; more simulations can be found in Van Groesen et al. (2007). To see the dependence of the results on simulation tools, we used as wave model both the well-known shallow water equations and a dispersive wave model, and we investigated the effect of the gridsize. Both wave models and all gridsizes will show qualitatively the same result that is illustrated in Fig. 1.

The dispersive simulations use a recently derived Variational Boussinesq model (VBM), and show some characteristic differences with simulations that use the shallow-water equations (SWE). Our results, partly summarised in Table 2, show that dispersive effects are clearly present and influence amplification factors, even for the geometries and bathymetries used here for which dispersive effects are expected to be small. The development of a dispersive tail would intuitively lead to a decrease of the largest crest-height. But it turns out that "dispersive amplitude exchange" between the first negative and the successive second positive wave largely compensate this decrease, and even increases the crest-height when running above a flat bottom. We capture these and similar effects over the total period of the propagation by using the "maximal temporal crest-height" as a convenient quantity to investigate the wavefield and to compare between different wavemodels and between simulations with different gridsizes.

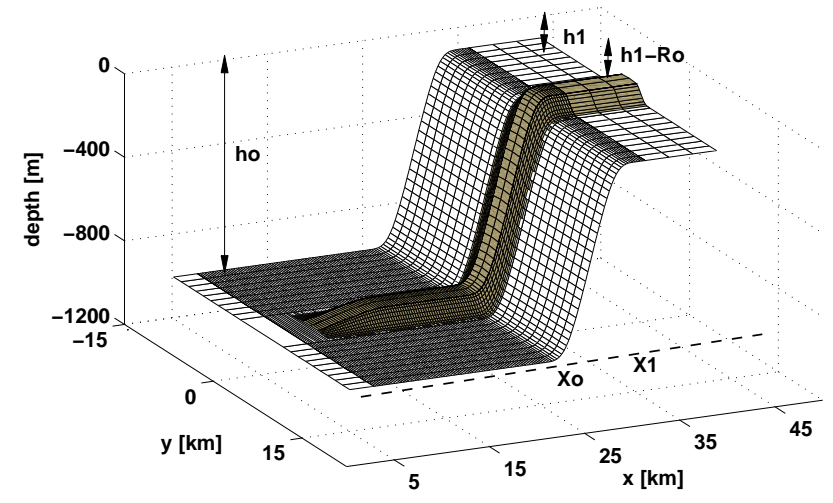

Fig. 2. Plot of the waveguiding bathymetry: the sloping bottom with the ridge that will act as waveguide.

Simulations with both wave models show a considerable dependence of the results on the gridsize that is used. This is caused by the fact that the physical process of tsunami waveguiding is largely determined by flow toward and from the waveguiding region. To capture that transversal flow sufficiently accurate, the gridsize should be sufficiently small. In the cases of the synthetic bathymetry that we will use in this paper, gridsizes of $250 \mathrm{~m}$ are required in both directions. When compared with gridsizes that are commonly used for simulations in off-coast regions, which are factors 6 to 8 times larger, the simulated distortion and amplification is much less and the whole process is severely delayed. The main findings are summarised in Table 3.

This observation may be the underlying explanation of the fact that tsunami waveguiding has not yet been reported as a possible (partial) explanation for large near-coast variability of waveheights, and consecutive effects on the coasts.

The organization of this paper is as follows. We comment on the various wave models in Sect. 2. In section 3 we specify and motivate the synthetic data for the initial tsunami profile and for the geometry and bathymetry of a coastal area with a waveguiding structure. In Sect. 4 we consider the simple problem of wave propagation over a flat bottom and in Sect. 5 the propagation toward the coast above a transversally uniform sloping bottom into shallow water. For the last case it is shown that the theoretical WKB results are close to the numerical results. In Sect. 6 we take this uniformly sloping bathymetry and add a ridge of fixed height that will act as waveguide. Various simulations will show the enhanced amplification of the crest-heights up to a factor of 2 to 3 . The dependence of the simulation results on the gridsize will be detailed in the final Sect. 7, together with some other aspects.

\section{Wave models and simulations}

In this section we briefly discuss the wave models that have been used for the simulations. 


\subsection{Shallow Water Models}

The linear and nonlinear wave models for shallow water waves, the so called Shallow Water Equations, are denoted by LSW and SW respectively in the following. These are the most commonly used models since the numerical implementations can deal well with the large domains that have to be considered and provide fast results for various implementations such as finite differences, finite elements and pseudospectral. For unidirectional propagation above flat bottom, wave profiles translate undisturbed in shape. When running into shallower water, part of the wave will be reflected. For the forth going wave, the speed and the wavelength decrease and the waveheight increases. These bathymetric induced changes are well described by the explicit theoretical expression known as the WKB-approximation, even when there is a substantial reflection, as we will see in Sect. 4 .

\subsection{The Variational Boussinesq Model}

The LSW and SW model ignore changes in the vertical direction in the fluid layer. Differences in surface elevation cause such variations to an extent that depends on the shallowness. The vertical variations lead to the effect of dispersion, which means that harmonic waves of smaller wavelength propagate slower than waves of larger wavelength. Since any initial surface elevation on a bounded area will contain harmonic waves of different wavelength, the time evolution of the initial disturbance will show the separation of the constituent waves. For a characteristic tsunami bipolar initial profile the result will be that a "dispersive tail" consisting of slower, smaller amplitude waves will become visible. The development of the tail leads to a decrease of the initial wave height. However, as we shall see, the entrance in still water may compensate this.

It is commonly argued that for tsunami-type of waves the dispersive effects can be neglected since such waves have initial wavelength of at least, say, $50 \mathrm{~km}$ when generated in the ocean where the depth is, say, $4000 \mathrm{~m}$. The initial shallowness ratio 4/50 will become only smaller when running into shallower water, proportional to the square root of the depth ratio. Nevertheless, as we will see from simulations in this paper, dispersion causes differences for tsunamis running above a flat bottom, a uniformly sloping bottom and when a waveguide is present.

The results of simulations to be presented were obtained using a pseudo-spectral implementation of a new variational Boussinesq model (VBM), see Klopman et al. (2005, 2006, 2007) for more details; we will refer to the linear version as LVB in the following. Just like any Boussinesq model, VBM approximates the vertical variations and leads eventually to a set of equations on the surface, hence a spatial reduction to 2HD. The VBM achieves this without introducing high order derivatives in the dynamic equations for the surface elevation $\eta$ and the scalar velocity potential $\phi$. However, these dynamic equations include an additional scalar variable $\psi$ which satisfies a linear elliptic equation that depends on the dynamic variables. For this combined set of equations, the elliptic equation has to be solved at each timestep.

The complete dynamics is derived in a consistent way from the variational formulation of the exact surface wave equations; these equations are a Hamiltonian system, with the total energy as Hamiltonian. To arrive at VBM, we first approximate the fluid potential by its value at the free surface plus a vertical potential profile that is multiplied by the spatially dependent variable $\psi$. Restricting the (kinetic) energy functional to such potential functions, the functional depends on the three $2 \mathrm{HD}$ quantities only. The best approximation is then obtained by minimizing with respect to $\psi$, leading to the elliptic equation. The dynamic equations are the Hamiltonian equations for this restricted functional. For the simulations in this paper, we used a vertical parabolic profile that satisfies the impermeability conditions on the varying bottom.

\section{Initial wave, geometry and bathymetry}

In the following we will take a specific quantitative example of a tsunami approaching the coast with a waveguide in the propagation direction. The choice of quantitative parameters in the simulations is motivated by the example of a tsunami of wavelength $40 \mathrm{~km}$ generated by tectonic plate motions at a depth of $4000 \mathrm{~m}$.

\subsection{Initial wave profile}

We consider propagation in the direction of the coast, taken as the $\mathrm{x}$-axis. At the generation region at a depth of $4000 \mathrm{~m}$ the wave has wavelength $\Lambda_{4000}=40 \mathrm{~km}$, and is uniform in the transversal (y-) direction. The period of this wave is $T=200 \mathrm{~s}$. To reduce the calculation domain, we start the simulation as if the wave has traveled without dispersion till a depth of $1000 \mathrm{~m}$. Neglecting the dispersion in this area will affect the rest of the calculations; a dispersive calculation over the full physical domain would show more dispersive effects, as is illustrated in Figs. 3 and 4.

For the initial wave profile we take a single period (smoothened) bipolar profile of the form $\partial_{x}\left(\cos ^{4}(x)\right)$, extended by zero outside the interval $(-\pi / 2, \pi / 2)$ and scaled to get the desired amplitude and wavelength.

To simulate that the initial wave elevation is caused by a fast bottom excitation of that form, we assume that this initial wave profile is released without speed; then this profile falls apart above a flat bottom in a right and left traveling wave of the same amplitude, illustrated in Fig. 3.

\subsection{Bathymetry}

We are interested in a sloping bottom toward the coast $(x)$ with a waveguiding structure in the transversal $(y)$ direction. We describe the details below. 


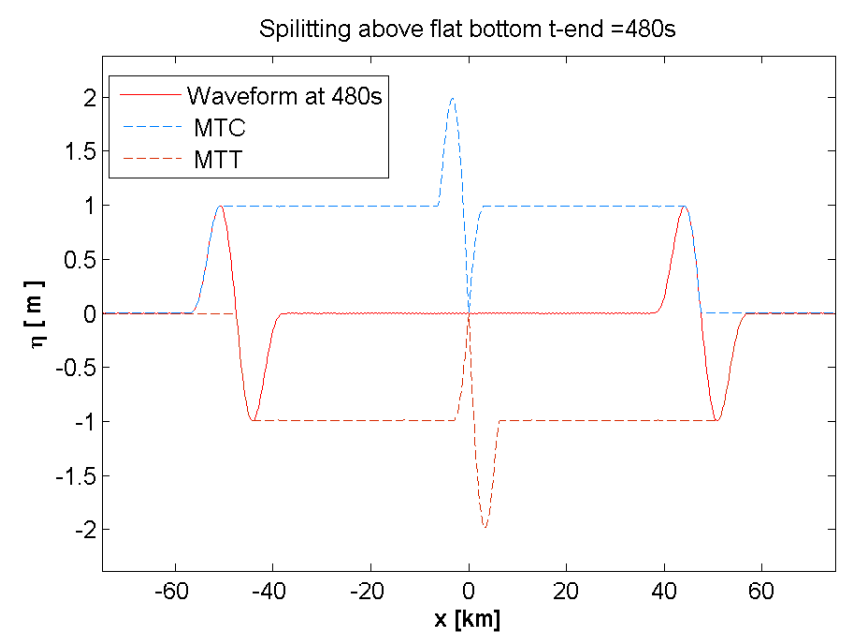

Fig. 3. For LSW-evolution is shown the initial splitting above a horizontal bottom in right and left traveling waves of half the initial amplitude. The dashed MTC and MTT curves show the unperturbed translation of the separated waves; at the end of the simulation time the wave is given by the solid line.

\subsubsection{Uniformly sloping bottom}

The bottom profile that is uniform in the transversal direction is described by a function $h_{u}(x)$. The bottom connects a depth $h_{0}$ for $x<X_{0}$ with a flat bottom region of smaller depth $h_{1}$ downstream for $x>X_{1}$. The depth is taken constant $h_{0}$ to the left to prevent possible reflection from bathymetry at the left. A smooth transition with average slope $\left(h_{0}-h_{1}\right) /\left(X_{1}-X_{0}\right)$ in the interval $x \in\left[X_{0}, X_{1}\right]$ is taken as follows

$h_{u}(x)=h_{0}+\left(h_{1}-h_{0}\right) \cdot H\left(\left(x-X_{0}\right) /\left(X_{1}-X_{0}\right)\right)$

where the smoothened transition function $H$ is 0 for $x<0$ and 1 for $x>1$ with transition $H(x)=\left[1+\tanh \frac{x-1 / 2}{x-x^{2}}\right] / 2$ for $0<x<1$.

\subsubsection{Waveguide geometry}

The waveguiding structure is created by a transversal depth variation that describes a shallow part in between deeper areas. For the simulations we consider a symmetric ridge of height $R_{0}$ with sloping sides. In the transversal direction we take the width at half-height to be $4 \mathrm{~km}$, with a flat top of width $2 W_{0}=2.5 \mathrm{~km}$ and sloping sides of width $S=1.5 \mathrm{~km}$, given by the function

$$
Y(y)=H\left(\left(y+W_{0}+S\right) / S\right)-H\left(\left(y-W_{0}\right) / S\right) .
$$

To prevent reflections of the incoming wave at the start of the wave guide, we let the ridge height increase smoothly from 0 at $x=X_{0} / 4$ till $R_{0}$ at $x=3 X_{0} / 4$. Taking together, the total waveguide structure is given by

$$
R(x, y):=R_{0} \cdot Y(y) \cdot H\left(\left(x-X_{0} / 4\right) /\left(X_{0} / 2\right)\right) .
$$

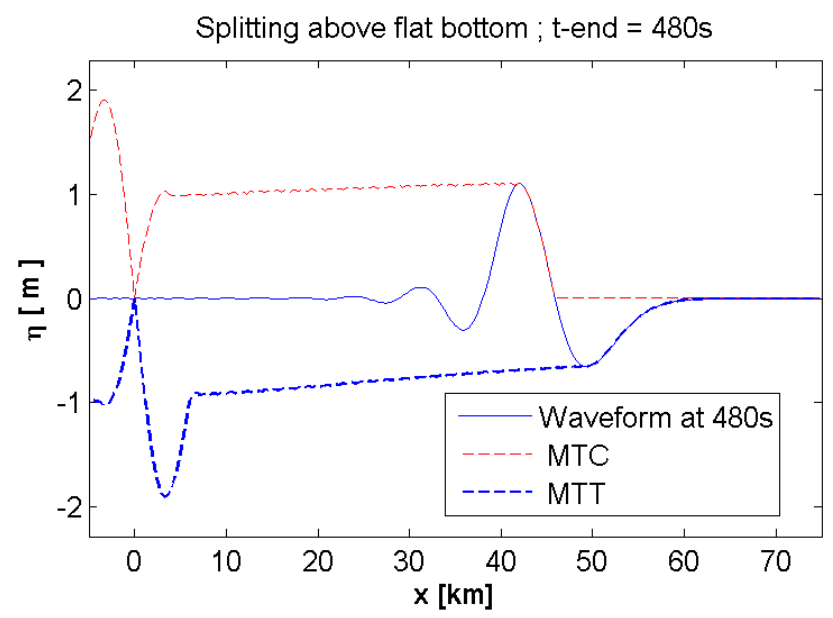

Fig. 4. LVB-simulation to show the effect of dispersion during propagation above flat bottom as in Fig. 3; here only the part of the wave running to the right is displayed.

\subsubsection{Waveguide on sloping bottom}

The waveguiding phenomenon will be illustrated for the bathymetry that is the uniform sloping bottom with the transversal ridge wave guide:

$h(x, y)=h_{u}(x)-R(x, y)$.

A schematic 3-D plot of the bathymetry is shown in Fig. 2.

\section{Evolution above flat bottom}

To illustrate the effect of dispersion in the simplest case, and to introduce how we will capture the propagation history, we start with the simple case of propagation above a flat bottom; see also Carrier and Yeh (2005). The initial wave with wavelength $\Lambda=20 \mathrm{~km}$ is released above the horizontal bottom at depth $1000 \mathrm{~m}$. The resulting evolution along the $\mathrm{x}$ axis is shown in Fig. 3 for non-dispersive evolution according to the LSW-model. It illustrates the splitting of the initial wave into a right and left traveling wave. In the plot we see the elevation at the end of the calculation time interval as the solid line. The dashed lines show some basic elements of the past dynamics; we will use these curves, to be abbreviated by MTC and MTT curve, in similar plots in the following. These MTC and MTT curves are variants of the maximal temporal amplitude introduced in Andonowati and Van Groesen (2003). Here they are the maximal temporal crest-height and minimal temporal trough-depth respectively, also called the maximal positive and negative amplitude respectively. At a position $x$, they represent the maximal and minimal surface elevation as function of time

$\operatorname{MTC}(x)=\max _{t} \eta(x, t), \operatorname{MTT}(x)=\min _{t} \eta(x, t)$. 


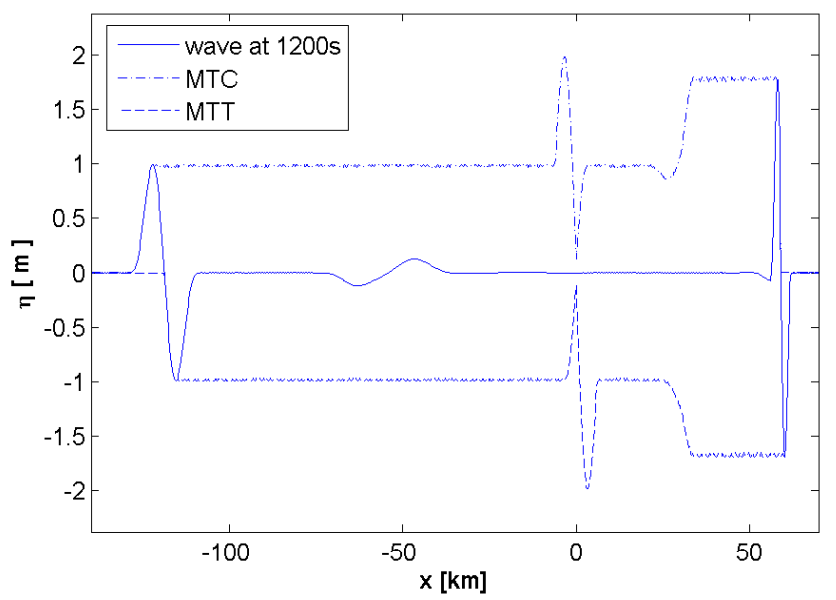

Fig. 5. LSW-simulation. To the left the wave is running above a flat bottom at depth $1000 \mathrm{~m}$, to the right there is a uniform run-up from $1000 \mathrm{~m}$ to $100 \mathrm{~m}$ depth between $25 \mathrm{~km}$ and $35 \mathrm{~km}$. Observe the reflected wave, traveling to the left.

The effect of dispersion is shown with a LVB-simulation using the MTC and MTT curves, displayed in Fig. 4. The bipolar profile changes as the combined effect of confinement of the initial profile and running into still water. We see that for the right running wave the negative wave elevation in front is reduced while the crest-height of the consecutive positive elevation becomes slightly larger. We will call this "dispersive amplitude exchange" caused by running into still water. Besides this we observe that a dispersive tail develops behind the bipolar wave.

\section{Uniform run-up}

We present in this section results for a uniform sloping bottom. For the numerical calculations we take the geometry described by $h_{u}(x)$ with parameter values $X_{0}=25 \mathrm{~km}$, $X_{1}-X_{0}=10 \mathrm{~km}$, and $h_{0}=1000 \mathrm{~m}$. We will consider three cases of depth after run-up: $h_{1}=100 \mathrm{~m}, h_{1}=60 \mathrm{~m}$ and $h_{1}=20 \mathrm{~m}$; these choices are based on the cases to be considered in the next section for waveguided run-up. As before, the initial wave has wavelength $\Lambda_{1000}=20 \mathrm{~km}$. For the simulations we used a gridsize of $250 \mathrm{~m}$ in each direction. This small gridsize is not needed for these simulations, but is taken for comparison with the waveguided run-up in the next section for which the choice of this small grid is essential.

\subsection{Simulation results}

Figure 5 shows results of simulations for the LSW-model; the wave evolution after $1200 \mathrm{~s}$ is given by the solid line, and the dashed lines are the MTC and MTT curves. In this simulation, to the left $(x<0)$ the wave is running over a flat bottom at depth $1000 \mathrm{~m}$, and to the right $(x>0)$ the wave is runningup till a depth of $100 \mathrm{~m}$. In Fig. 6 we show the similar plot

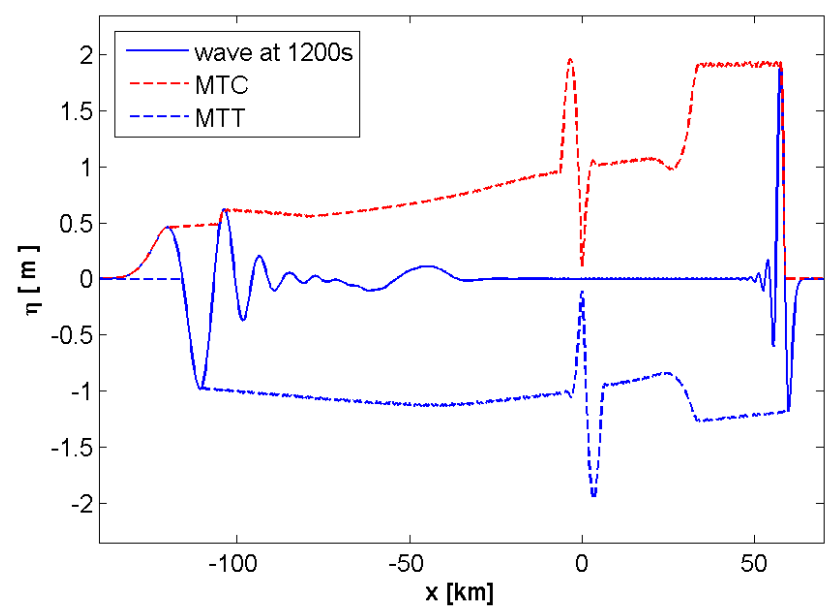

Fig. 6. For LVB-simulation, the same case as Fig. 5.

for the dispersive dynamics with the LVB-model. For this last case, a 2-D and a 3-D image is given for the wave after run-up in Fig. 7.

\subsection{Discussion of simulation results}

We briefly comment here on the results for uniform run-up.

\subsubsection{Qualitative observations}

All results show clearly the well-known property that during run-up the crest-height increases and that the wavelength and the propagation speed decrease, very close to predictions by the WKB-method, as we shall see below. The simulations show a substantial reflection from the run-up region, visible in the left traveling wave near position $-50 \mathrm{~km}$.

The difference between the non-dispersive LSW and the dispersive LVB-model is the distortion of the wave form: the LSW-evolution retains almost the skew-symmetric profile, while the LVB evolution shows a substantial dispersive exchange of amplitude between the front and consecutive wave, and the development of a dispersive tail.

\subsubsection{Quantitative results}

For a uniformly sloping bathymetry, the run-up is essentially one-dimensional and we can use simple expressions for wavelength and amplitude changes according to the LSWmodel. The wave speed $c$ is then related to the depth $h$ by $c=\sqrt{g h}$, and the WKB-approximation (see Van Groesen and Molenaar, 2007) describes the corresponding wavelength and amplitude changes, under the assumption of mildly sloping bathymetries. The wavelength $\Lambda$ decreases with the square root, and the amplitude increases with the fourth root of the depth:

$$
\Lambda(h)=\Lambda\left(h_{0}\right) \sqrt{h / h_{0}} \quad \text { and } \quad a(h)=a\left(h_{0}\right) \sqrt[4]{h_{0} / h} .
$$



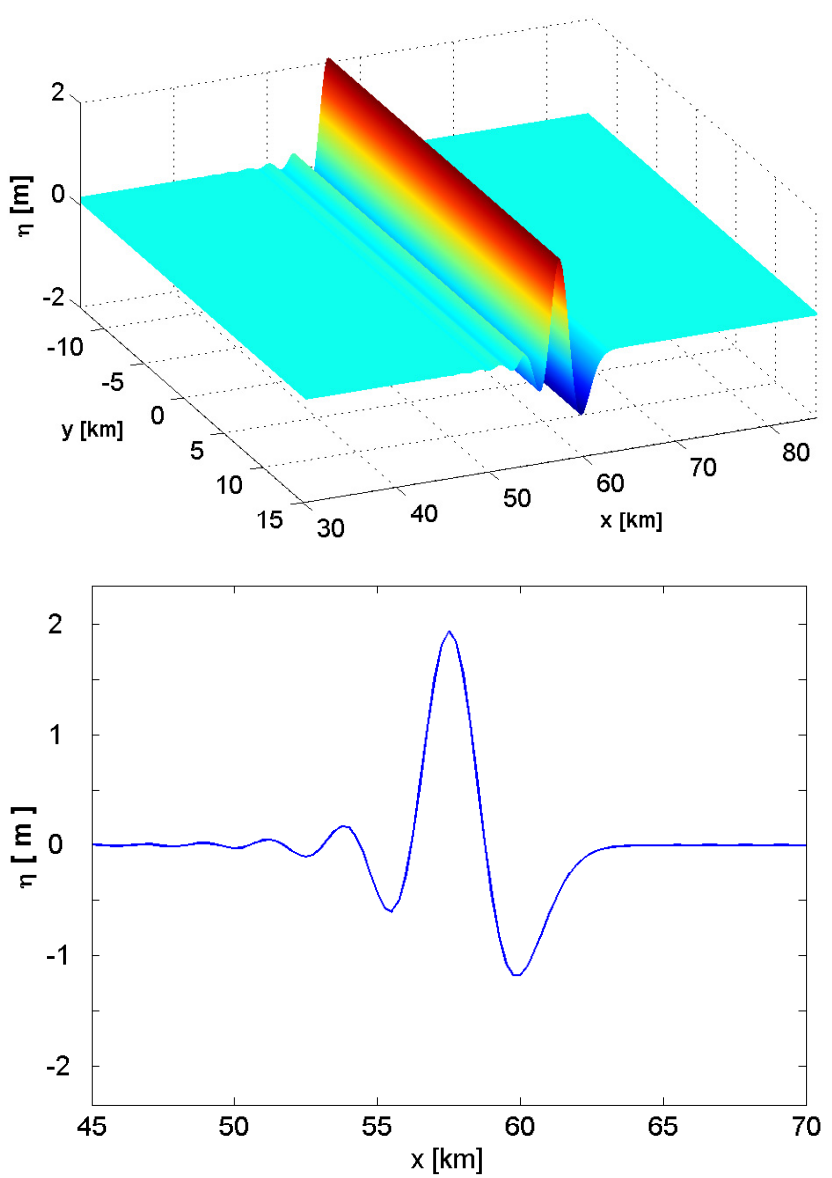

Fig. 7. LVB-simulations of uniform run-up, with 2-D and 3-D plots of the wave after run-up.

Table 1. Table of amplitude amplification for wave run-up from 1000 m depth till depth $h_{1}$.

\begin{tabular}{cccc}
\hline depth $h_{1}$ & WKB & LSW & LVB \\
\hline $100 \mathrm{~m}$ & 1.8 & 1.9 & 1.9 \\
$60 \mathrm{~m}$ & 2.0 & 2.0 & 2.2 \\
$20 \mathrm{~m}$ & 2.7 & 2.8 & 2.8 \\
\hline
\end{tabular}

In Table 1 the maximal crest-heights (immediately) after run-up are given for the theoretical WKB approximation and for the numerical results for LSW- and LVB-evolution for three cases of run-up. All numerical values are close to the theoretical WKB-approximation. This is actually somewhat remarkable if we take into account that the bottom steepness is not particularly small (slope $\approx 1: 11$ ), as was evidenced by the substantial large reflection. This observation about the applicability of the WKB results is in line with Synolakis (1991).

\section{Waveguided run-up}

In this section we investigate the run-up when a waveguide is present. For the numerical calculations we take the same initial profile as before. The bathymetry is given by $h_{u}(x)-R(x, y)$ with parameter values $X_{0}=25 \mathrm{~km}$, $X_{1}-X_{0}=10 \mathrm{~km}$, and $h_{0}=1000 \mathrm{~m}$. We will consider two cases of depth after run-up corresponding to two cases of ridge height: $R_{0}=40 \mathrm{~m}$ and $R_{0}=80 \mathrm{~m}$, so that for $x>X_{1}$ the depth above the waveguide is $60 \mathrm{~m}$ and $20 \mathrm{~m}$ respectively, surrounded by a depth of $100 \mathrm{~m}$.

As a measure of the strength of a waveguide we introduce the "relative index" $N$ as the quotient of the velocities outside and inside the waveguide. For the waveguided run-up this index is then given by

$N=\sqrt{h_{u} /\left(h_{u}-R_{0}\right)}$.

Since the exterior depth decreases while the ridgeheight remains constant, the strength will increase during run-up. For $R_{0}=40 \mathrm{~m}$ the strength increases from $N=1.02$ at $x=X_{0}$ to $N=1.29$ for $x>X_{1}$, while for $R_{0}=80 \mathrm{~m}$ the strength increases from $N=1.04$ at $x=X_{0}$ to $N=2.24$ for $x>X_{1}$.

For the simulations we used a gridsize of $250 \mathrm{~m}$ in each direction. This choice of a relatively small gridsize was made to assure that in the longitudinal and transversal direction all aspects of the waves are covered well. In the longitudinal direction, at the most shallow depth of $20 \mathrm{~m}$ that we will encounter in the simulations, the wavelength of nondispersive waves would be approximately $20 / 7 \mathrm{~km}$, so that there are some ten grid points in the waves of smallest wavelength. In the transversal direction, this small gridsize turned out to be necessary to simulate correctly the flow toward and from the waveguide; see Sect. 7 for remarks about simulations with coarser grids.

A numerical window of large width of $30 \mathrm{~km}$ around the waveguide of $4 \mathrm{~km}$ in the transversal direction had to be taken to assure that at the numerical boundary the run-up wave did not experience any disturbance by the presence of the waveguide.

\subsection{Simulation results for tsunami waveguiding}

The symmetry in the profiles that we found causes the maximal heights to appear at the center of the waveguide, at $y=0$. In fact, for the given width of the waveguide, we only observed the "fundamental" mode, which is symmetric. For wider waveguides, higher order modes were also found. Therefore, we plot the wave evolution in the coastal direction by using the MTC and MTT curves at the cross section $y=0$. For the two cases $R_{0}=40 \mathrm{~m}$ and $R_{0}=80 \mathrm{~m}$ we show some results of simulations with the LSW- and LVB-models in Figs. 8 and 9.

Although the initial wave is uniform in the transversal ydirection, the presence of the waveguide will disturb the uniformity. We present some density and 3-D plots to illustrate 

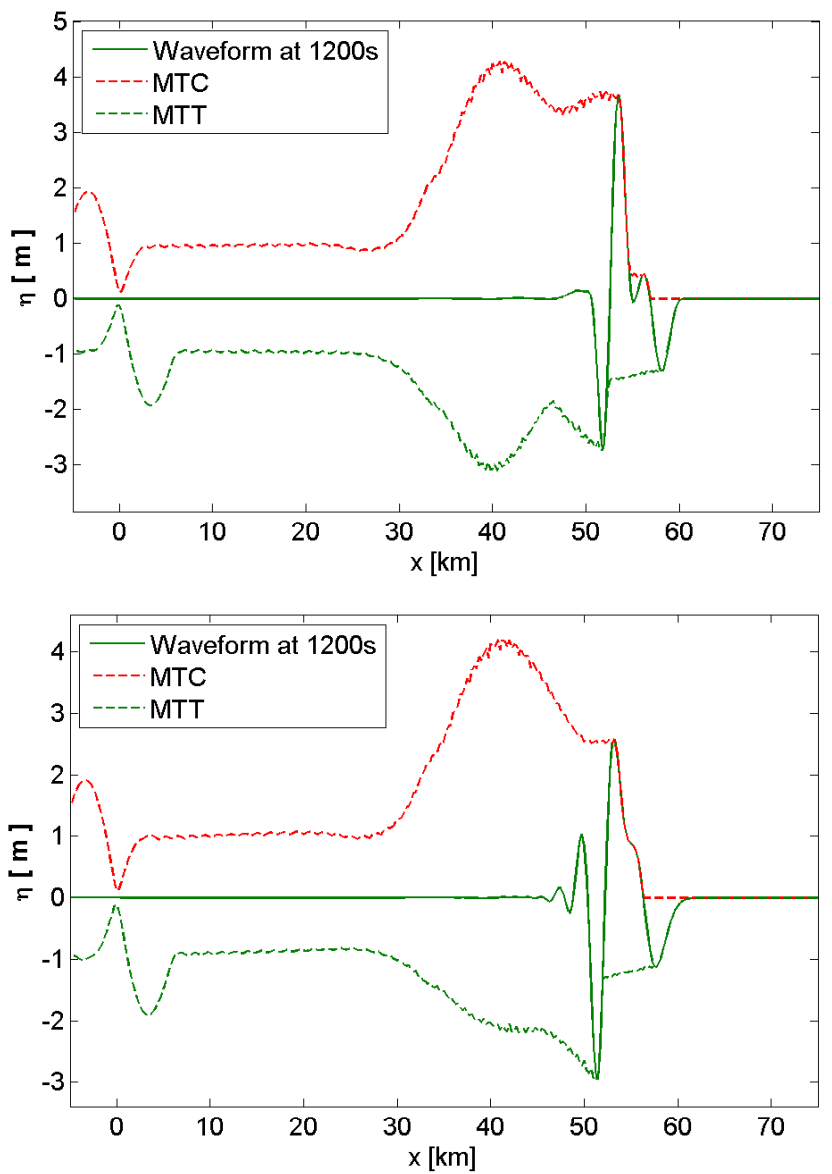

Fig. 8. Tsunami waveguiding for ridge height $R_{0}=40 \mathrm{~m}$; above for the LSW-model and below for the LVB-model.

the deformation in a global way, and then show transversal cross-sections in more detail for the case of the waveguide with $R_{0}=40 \mathrm{~m}$, in Figs. 10, 11, and 12.

While running further downstream, the wave disintegrates and develops a more pronounced tail while the crest-height decreases; Fig. 13 shows some characteristics at the end of the calculation interval of $1200 \mathrm{~s}$.

\subsection{Discussion about simulation results}

\subsubsection{Qualitative observations}

The most dominant phenomenon of tsunami waveguiding is visible in all simulations: the substantial enlargement and delay of the wave above the waveguide compared to the transversal surrounding wave.

During the waveguiding, the waveform changes considerably; the wave of highest crest-height is found at or after the end of the run-up region. The profile has a substantially reduced negative wave in the front, a very much enlarged consecutive positive wave and a consecutive negative wave, which is deeper for LVB than for LSW. The deformation also
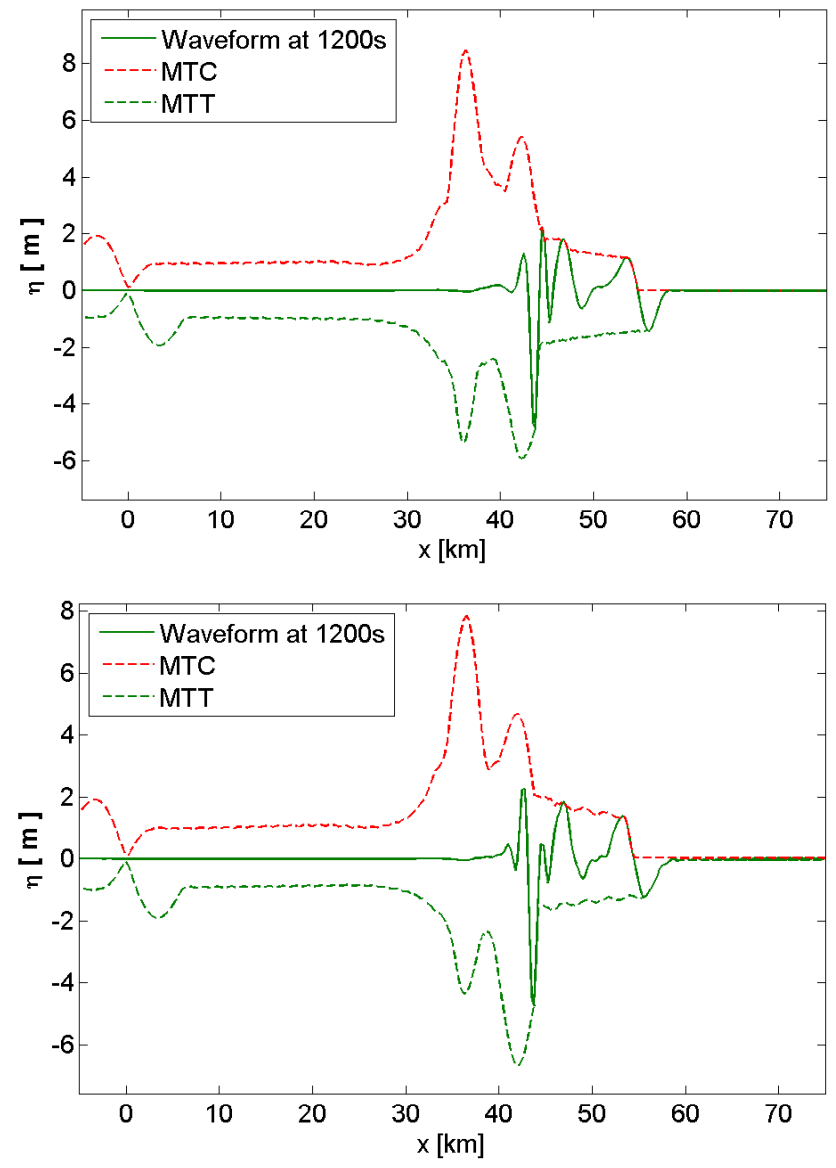

Fig. 9. Tsunami waveguiding for $R_{0}=80 \mathrm{~m}$ for the LSW- (above) and LVB-model (below).

happens for the LSW-evolution, different than in case of uniform run-up. The crest-height and waveheight are very large compared to the depth in case $R_{0}=80 \mathrm{~m}$; the waveheight is more than half the depth. Yet, because the wavelength is still relatively large, the waves are not steep and vertical motions are still rather small.

The transversal deformations are considerable, caused by the delay of the wave above the waveguide. The density plots and the transversal sections show that transversal deformations extend till 4 times the width of the waveguide (for the time interval under consideration). Outside that area, the wave adjusts to the surrounding wave that runs-up uniformly undisturbed till the depth $100 \mathrm{~m}$. This implies that there it propagates with the speed determined by the exterior depth, and hence that the wave as a whole during run-up travels with that speed. This is confirmed by the numerical simulations: with or without the presence of the waveguide, the progress of the front of the wave is almost the same. The transversal variations are the main reason why the discretization in the transversal direction has to be sufficiently small and has to be extended sufficiently far. 

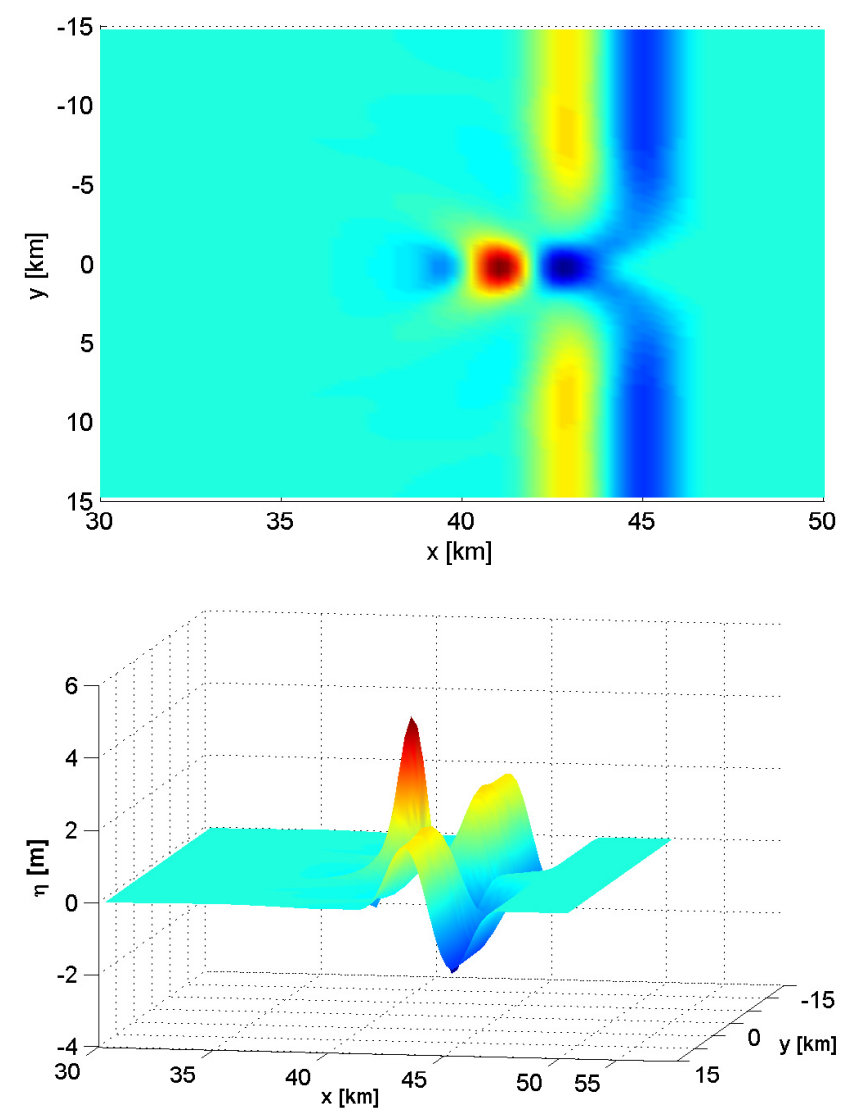

Fig. 10. Tsunami waveguiding with LSW-evolution for $R_{0}=40 \mathrm{~m}$; snapshots at the time of maximal crest-height $(t=720 \mathrm{~s})$ of the wave, above a density plot and below a 3-D impression.

Table 2. Amplification and enhancement factors for the maximal crest-height.

\begin{tabular}{ccccc}
\hline$R_{0}$ & wave model & TWG & $Q_{\text {ext }}$ & $Q_{\text {wg }}$ \\
\hline $40 \mathrm{~m}$ & LSW & 4.3 & 2.4 & 2.1 \\
$40 \mathrm{~m}$ & LVB & 4.2 & 2.2 & 1.9 \\
$80 \mathrm{~m}$ & LSW & 8.5 & 4.7 & 3.1 \\
$80 \mathrm{~m}$ & LVB & 7.8 & 4.1 & 2.8 \\
\hline
\end{tabular}

Having arrived at the flat waveguided area, the distorted wave seems to adapt to the horizontal waveguide. In the cases shown, initially the crest-height decreases, after which further deformations (shown in the plots of the profile at the endtime, Fig. 13) determine the details.

\subsubsection{Summary of quantitative results}

In Table 2 we summarize the quantitative results for the maximal crest-height. In the first column the height of the waveguide is given; the second column describes the wave
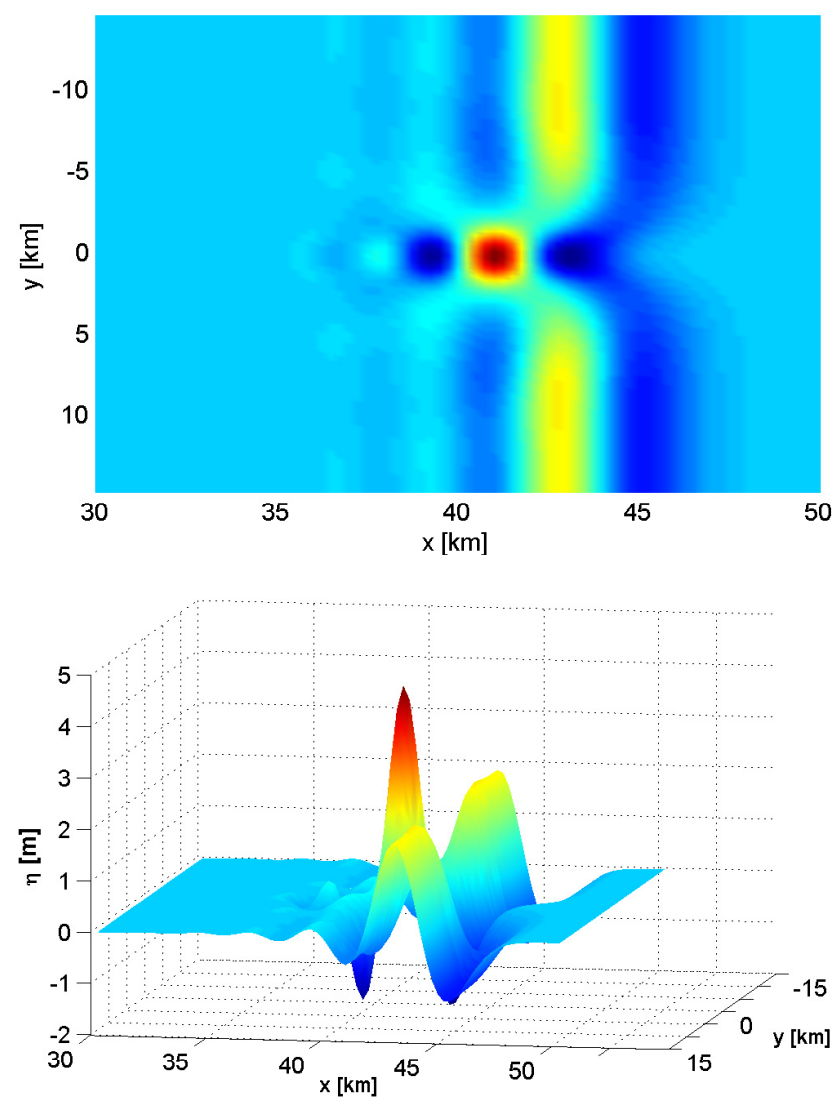

Fig. 11. Tsunami waveguiding with LVB-evolution for $R_{0}=40 \mathrm{~m}$ as in Fig. 10.

model. The total amplification is given in column 3 , where we take the maximal crest-height (compared to the initial crest height) as measure. For comparison with the crestheight increase for uniform run-up, the quotient of the maximal amplitudes with and without the presence of the waveguide, the waveguide-enhancement, is denoted by $Q$. Depending on whether we take the exterior depth or the depth above the waveguide as reference, and using the results listed in Table 1, we get the values of the quotients $Q_{\text {ext }}$ and $Q_{\text {wg }}$ as given in columns 4 and 5 .

\section{Discussion}

In this paper we presented results of numerical simulations that convincingly show the phenomenon of tsunami waveguiding. To our best knowledge, this phenomenon did not receive attention yet in the literature. In this final section we comment on various different aspects. 

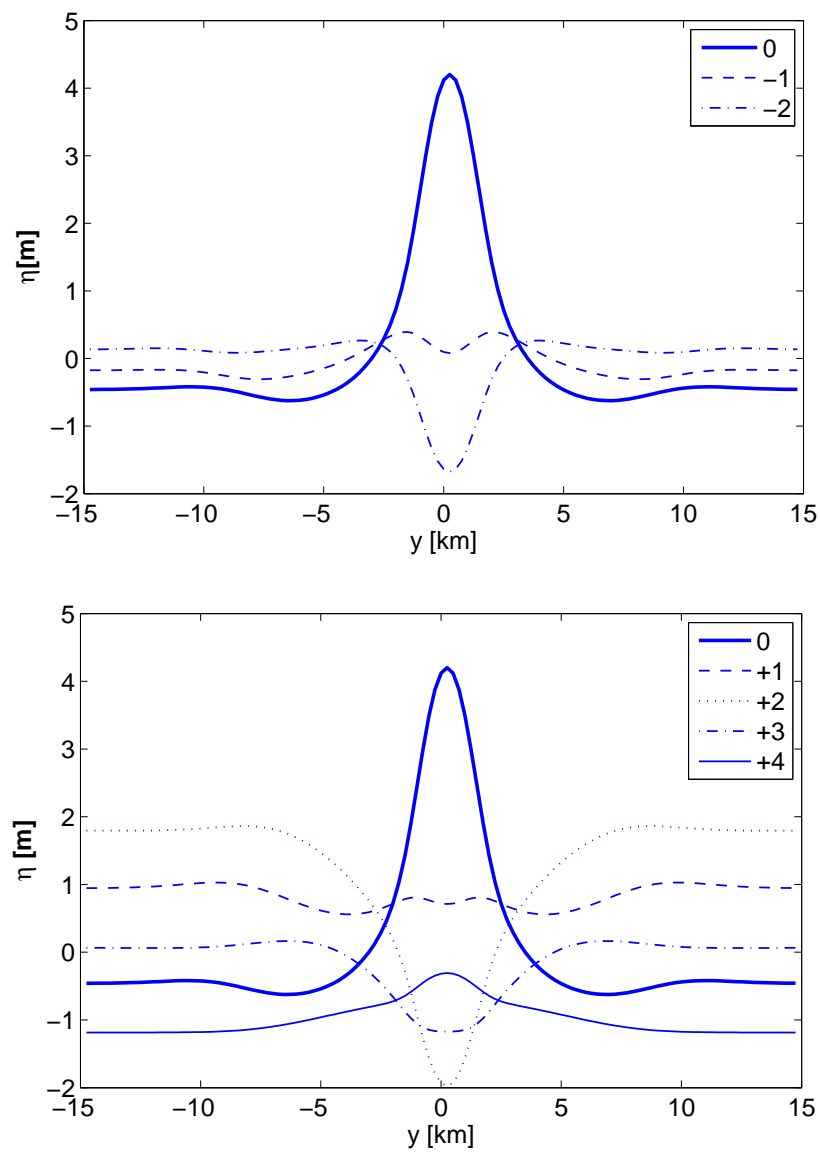

Fig. 12. For LVB-evolution and $R_{0}=40 \mathrm{~km}$, transversal crossections (y-axis horizontal) show the waveprofile of maximal crestheight (at $t=720 \mathrm{~s}, \mathrm{x}=41 \mathrm{~m}$, denoted by 0 ) and the profiles at the same instant 1 and $2 \mathrm{~km}$ behind $(-1,-2$ in above plot) and 1, 2, 3 and $4 \mathrm{~km}$ ahead $(+1, \ldots,+4$ in lower plot $)$ of the extreme position.

\subsection{Gridsize dependence}

The reduced speed of the wave above the waveguide causes transversal flows which lead to large amplification. This is a subtle process that may influence the accuracy of simulations (see also Liu, 2005). To investigate this, we performed calculations on gridsizes that are larger than used in the rest of this paper. Assuming that the waveguide at the shallow flat part after run-up continues, we found the results as listed in Table 3. In that table, the maximal crest-height is given together with the position and time of occurrence of the maximal crest-height. It shows that the maximal crest-height is lower for coarser grids, and in particular that the process of amplification seems to be delayed considerably. These results indicate that the grids that are often used in tsunami calculations at deeper parts of the ocean, $1^{\prime} \times 1^{\prime} \approx 1850 \mathrm{~m} \times 1850 \mathrm{~m}$, are too coarse and should be refined to capture tsunami waveguiding before but close to the coast.
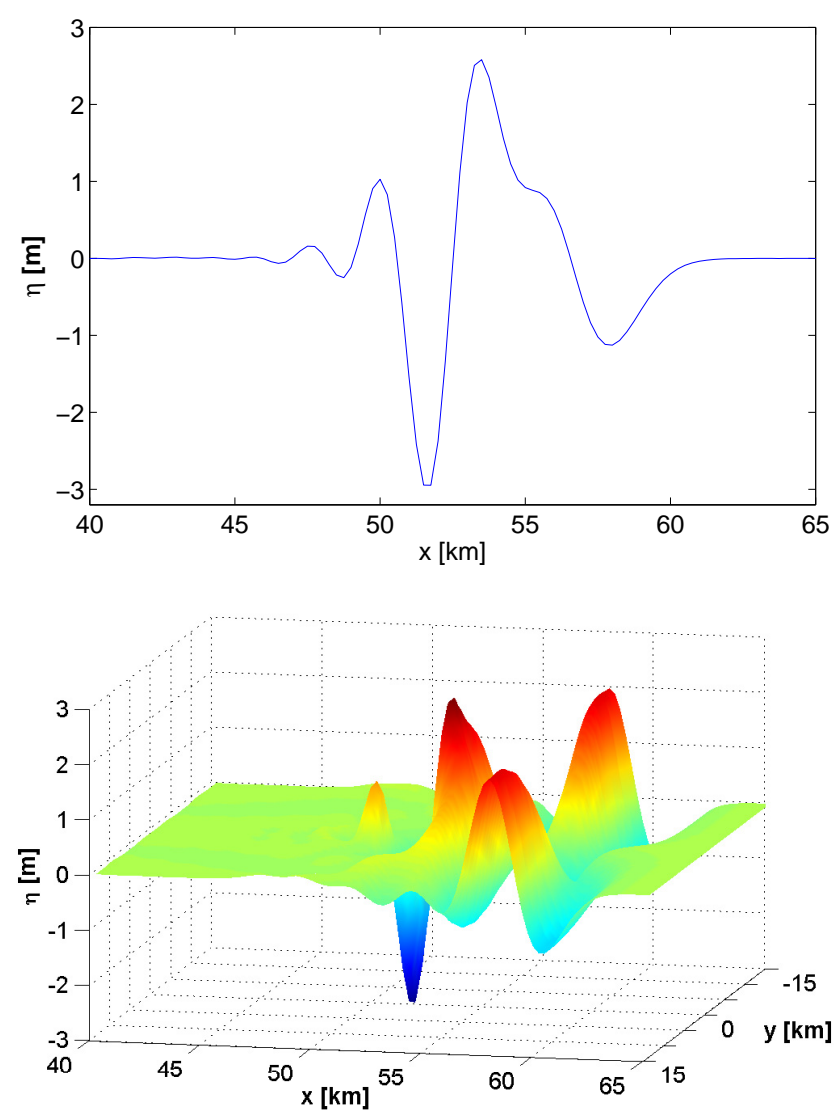

Fig. 13. For LVB-evolution and $R_{0}=40 \mathrm{~m}$, the waveprofile in the propagation direction at the centreline (above) and a 3-D impression (below) at the end time $1200 \mathrm{~s}$ of the simulation.

Table 3. Maximal crest-height with position and time of occurrence.

\begin{tabular}{cccc}
\hline Grid $(\mathrm{m} \times \mathrm{m})$ & Max crest-height $(\mathrm{m})$ & Position $(\mathrm{km})$ & Time $(\mathrm{s})$ \\
\hline $250 \times 250$ & 4.3 & 41 & 720 \\
$500 \times 500$ & 3.8 & 54 & 1275 \\
$1000 \times 1000$ & 3.7 & 61 & 1395 \\
\hline
\end{tabular}

\subsection{Reversed wave}

The choice of the initial wave is motivated by tsunami waves that are generated by tectonic plate motions relatively close to the coast of interest. Then, in case of rupture, the subduction of the oceanic plate under the land plate causes a downward motion of the bottom, and hence a negative surface elevation at the landside and an upward motion with positive surface elevation at the ocean side. We will now investigate the so-called reversed wave, i.e. the wave traveling in the direction with the positive wave in front. Based on the symmetry in the (linear) equations, it can be expected that 

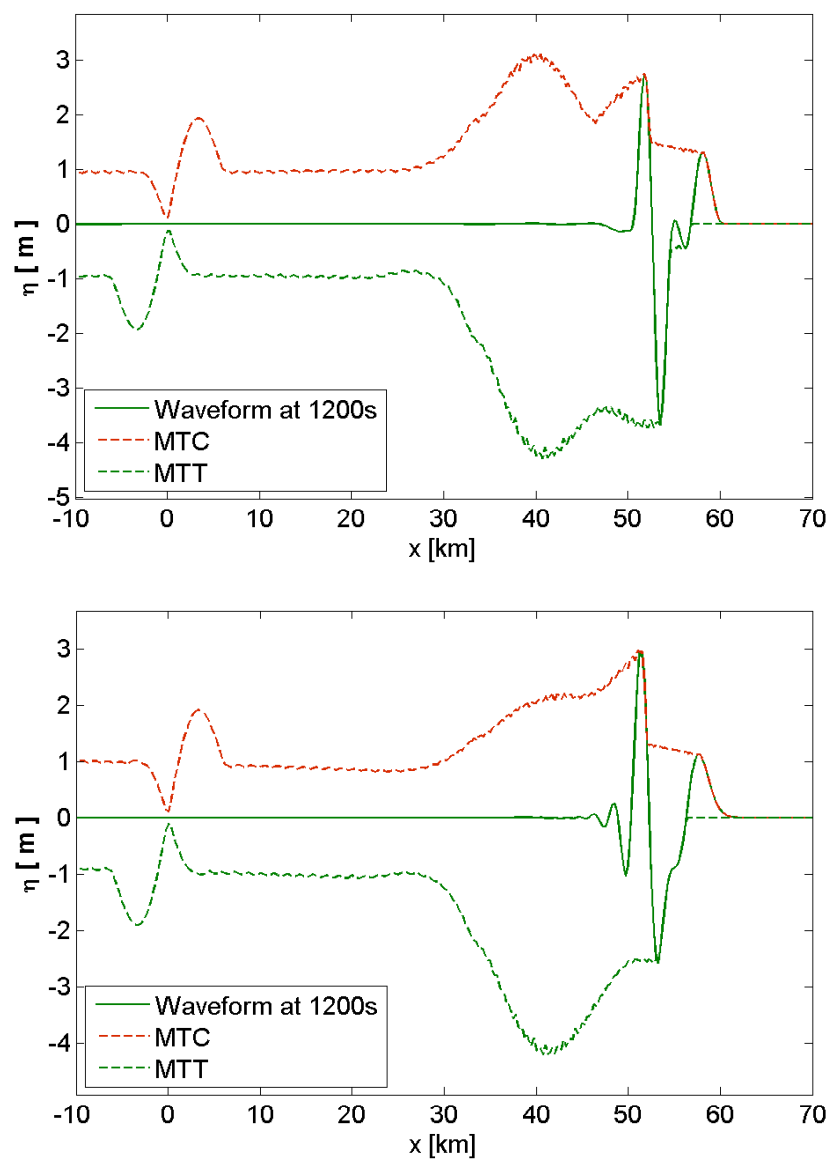

Fig. 14. Tsunami waveguiding for $R_{0}=40 \mathrm{~m}$ for the reversed wave, above for LSW- and below for LVB-simlation.

the same waveguiding phenomenon happens for the reversed wave. The following results of calculations for a reversed initial wave show that this is indeed the case: now the negative wavepart shows largest amplification, and the plots are almost the same for the corresponding case with opposite sign of elevation.

For LSW- and LVB-evolutions this can be seen by comparing the plots for the reversed wave Figs. 14, and 15 with the previous results Fig. 8 and 10; see also Kanoglu (2004).

\subsection{Choice of the synthetic data}

At this moment we are investigating data for regions where a waveguiding bathymetry is present in the Indonesian coastal area; the results will be published in a forthcoming paper. The synthetic data that we used in this paper are much simpler than realistic bathymetries; for that reason we briefly discuss the major aspects of the choices of the synthetic data.

A first remark in this respect concerns the observation that the invariance of the shallow wave equations imply that the results are robust and characteristic for a class of geometries and incoming waves. In fact, for any scaling factor $\alpha$ the in-
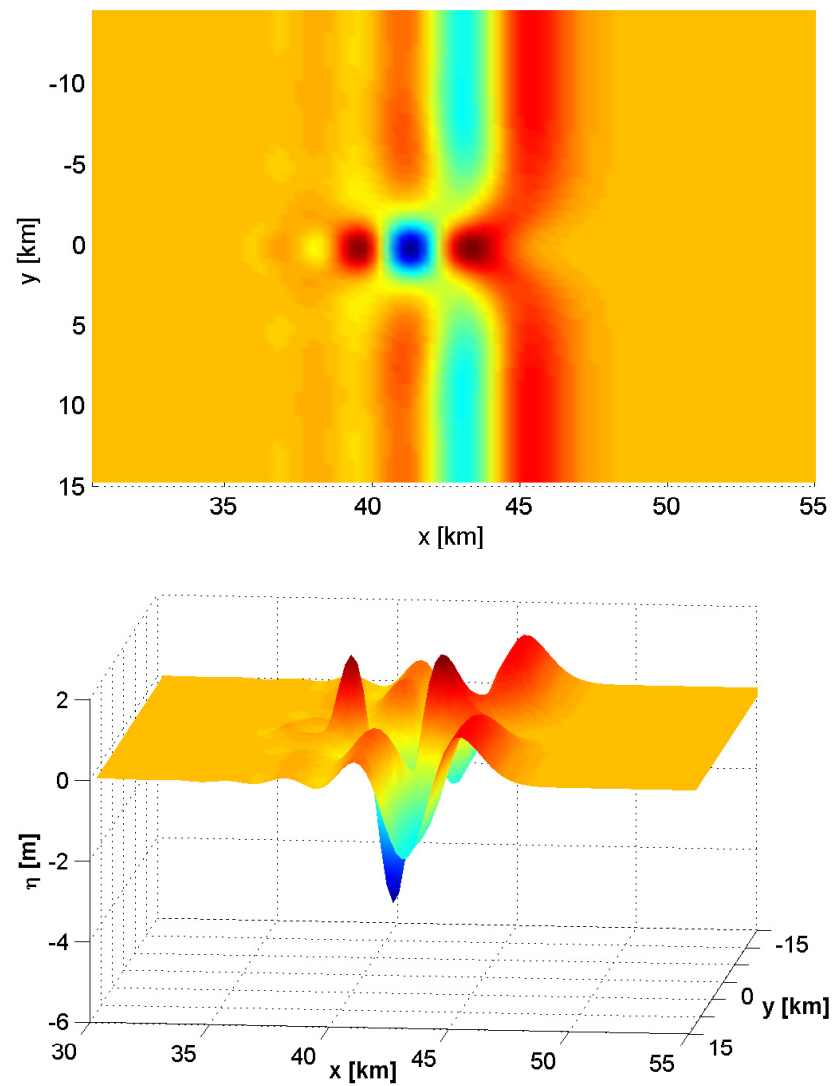

Fig. 15. Tsunami waveguiding for LVB and $R_{0}=40 \mathrm{~m}$ for the reversed wave.

variance is given by $(x, y, \omega) \rightarrow(x / \alpha, y / \alpha, \alpha \omega)$. If we take the depth of the generation of the tsunami fixed, the scaling in the frequency corresponds to a scaling of the initial wavelength $\Lambda \rightarrow \alpha \Lambda$. Hence, if the width of the waveguide is scaled similarly, $W \rightarrow W / \alpha$, the same wave amplification factors are found.

The choice of the specific waveguide is more problematic. As waveguiding structure we took here a simple ridge of fixed width and height on top of the sloping bottom. The assumption on the sloping bottom seems less severe than the choice of the (fixed) height of the ridge. This can be seen already by the different results for $R_{0}=40 \mathrm{~m}$ and $R_{0}=80 \mathrm{~m}$ ridge height. In both cases the entrance region has no noticeable effect since the strength of the waveguide at the deep bottom is small. The waveguided run-up, however, is very different. For $R_{0}=80 \mathrm{~m}$ the total amplification from $1 \mathrm{~m}$ to approximately $8 \mathrm{~m}$ takes place in the run-up region of $10 \mathrm{~km}$ length, and $1 \mathrm{~km}$ behind it. For $R_{0}=40 \mathrm{~m}$ the increase in the run-up region is approximately from $1 \mathrm{~m}$ to approximately $2.5 \mathrm{~m}$, but a substantial further increase is observed while running over an additional distance of $6 \mathrm{~km}$ over the horizontal part $x>X_{1}$. In both cases, after the increase a slow $\left(R_{0}=40 \mathrm{~m}\right)$ or fast $\left(R_{0}=80 \mathrm{~m}\right)$ decrease sets in, related to the development of a more profound tail. 
Acknowledgement. This work is part of Mobility Programme 05-MP-08 in the Scientific Collaboration Indonesia-Netherlands funded by KNAW (Royal Netherlands Academy of Arts and Sciences). We appreciated very much conversations with S. Widiyantoro and D. Natawijaya who gave valuable background knowledge about seismological aspects, and with A. Sopaheluwakan and with our partners in the Mobility Programme, N. S. Ningshi, A. Suryanto and M. Nurhuda about the numerical aspects of tsunami simulations. We acknowledge with pleasure the use of the pseudo-spectral implementation of the LVB-model by G. Klopman, and useful references suggested by the referees.

Edited by: S. Tinti

Reviewed by: two anonymous referees

\section{References}

Andonowati and Van Groesen, E.: Optical pulse deformation in second order nonlinear media, J. Nonlinear Optical Physics and Materials, 12, 221-234, 2003.

Borrero, J.C., Synolakis, C. E., and Fritz, H.: Northern Sumatra Field Survey after the December 2004 Great Sumatra Earthquake and Indian Ocean Tsunami, Earthquake Spectra, 22(S3), S93S104, 2006.

Carrier, G. and Yeh, H.: Tsunami propagation from a finite source, CMES Comp. Model. Eng., 10(2), 113-122, 2005.

Chatenoux, B. and Peduzzi, P.: Analysis of the role of bathymetry and other environmental parameters in the impacts from the 2004 Indian Ocean tsunami. Report for the UNEP Asian Tsunami Disaster Task Force, UNEP/DEWA/GRID-Europe, Switzerland, 2005.

EERI: Distribution of the Tsunami Heights of the 2004 Sumatra Tsunami in Banda Aceh measured by the Tsunami Survey Team, Earthquake Engineering Research Institute, available online at: http://www.eri.u-tokyo.ac.jp/namegaya/sumatera/ surveylog/eindex.htm, EERI Special Earthquake Report, 2005, The Great Sumatra Earthquake and Indian Ocean Tsunami of December 26, 2004, Earthquake Engineering Research Institute, Oakland, CA, 2005.

Fritz, H. M., Kongko, W., Moore, A., et al.: Extreme runup from the 17 July 2006 Java tsunami, Geophys. Res. Lett., 34, L12602, doi:10.1029/2007GL029404, 2007.

Fujima, K., Dozono, R., and Shigemura, T.: Generation and propagation of tsunami accompanying edge waves on a uniform sloping shelf, Coast. Eng. J., 42, 211-236, 2000.

Gonzalez, F. I., Satake, K., Boss, E. F., and Mofjeld H. O.: Edge wave and non-trapped modes of the 25 April 1992 Cape Mendocino Tsunami, Pure Appl. Geophys., 144, 409-426, 1995.

Ishii, H. and Abe, K.: Propagation of tsunami on a linear slope between two flat regions. I. Eigenwave, J. Phys. Earth, 28, 531541,1980

Kajiura, K.: Tsunami source, energy and directivity of wave radiation, Bull. Earthq. Res. Inst., 48, 835-869, 1970.
Kanoglu, U.: Nonlinear evolution and runuprundown of long waves over a sloping beach, J. Fluid Mech., 513, 363-372, 2004.

Klopman, G., Dingemans, M., and Van Groesen, E.: A variational model for fully non-linear water waves of Boussinesq type, Proceedings of 20th International Workshop on Water Waves and Floating Bodies, Spitsbergen, Norway, 29 May-1 June 2005, http://www.iwwwfb.org/Abstracts/iwwwfb20/iwwwfb20_ 30.pdf, 2005.

Klopman, G., Van Groesen, E., and Dingemans, M.: Variational Boussinesq modeling of fully nonlinear water, J. Fluid Mech., in review, 2006.

Klopman, G., Dingemans, M., and Van Groesen, E.: Propagation of wave groups over bathymetry using a variational Boussinesq model, Proceedings of 22th International Workshop on Water Waves and Floating Bodies, edited by: Malenica, Ł̌. and Senjanovi, I., Plitvice, Croatia, April 2007, 125-128, 2007.

Kurkin, A. and Pelinovsky, E.: Focusing of edge waves above sloping beach, Eur. J. Mech. B-Fluid, 21, 561-577, 2002.

Liu, P. L. F.: Tsunami Simulations and Numerical Models, National Academy of Engineering, 35, 2, http://www.nae.edu/NAE/ bridgecom.nsf/weblinks/MKEZ-6DFRPP?OpenDocument, 2005.

Liu, P. L., Fernando, H., Jaffe, B. E., Fritz, H., Higman, B., Morton, R., Goff, J., and Synolakis, C.: Observations by the International Tsunami Survey Team in Sri Lanka, Science, 308, p. 1595, 2005.

Reese, S., Cousins, W. J., Power, W. L., Palmer, N. G., Tejakusuma, I. G., and Nugrahadi, S.: Tsunami vulnerability of buildings and people in South Java field observations after the July 2006 Java tsunami, Nat. Hazards Earth Syst. Sci., 7, 573-589, 2007, http://www.nat-hazards-earth-syst-sci.net/7/573/2007/.

Synolakis, C. E.: Tsunami runup on steep slopes: How good linear theory really is, Nat. Hazards, 4, 221-234, 1991.

Synolakis, C. E. and Bernard, E. N.: Tsunami science before and beyond Boxing Day 2004, Phil. Trans. R. Soc. A, 364, 22312265, doi:10.1098/rsta.2006.1824, 2006.

Synolakis, C. E. and Kong, L.: Runup Measurements of the December 2004 Indian Ocean Tsunami, Earthquake Spectra, 22(S3), S67-S91, 2006.

Titov, V. V. and Synolakis, C. E.: Extreme inundation flows during the HokkaidoNansei-Oki tsunami, Geophys. Res. Lett., 24, 1315-1318, 1997.

Titov, V. V., Rabinovich, A. B., Mofjeld, H. O., Thomson, R. E., and Gonzalez, F. I.: The global reach of the 26 December 2004 Sumatra tsunami, Science, 309, 2045-2048, 2005.

Van Groesen, E. and Molenaar, J.: Continuum modeling in the Physical Sciences, SIAM Philadelphia, 2007.

Van Groesen, E., Adytia, D., Andonowati, and Klopman, G.: Near-coast tsunami waveguiding: simulations for various wave models, Technical Report of LabMath-Indonesia, LMIGeoMath-07/02, October 2007, ISBN 90-365-2352-4, http:// www.labmath-indonesia.or.id/Reports/Reports.php, 2007.

Yeh, H., Imamura, F., Synolakis, C., Tsuji, Y., Liu, P., and Shi, S.: The Flores Island tsunamis, EOS, Trans. Amer. Geophys. Un., 74, p. 369 and 371-373, 1993. 